\title{
Application of Support Vector Machine Classifiers to Preoperative Risk Stratification With Myocardial Perfusion Scintigraphy
}

\author{
Tomotaka Kasamatsu, MD; Jun Hashimoto, MD; Hitoshi Iyatomi, PhD*; \\ Tadaki Nakahara, MD; Jingming Bai, MD**; Naoto Kitamura, MD; \\ Koichi Ogawa, PhD*; Atsushi Kubo, MD
}

\begin{abstract}
Background Myocardial perfusion single-photon emission computed tomography (SPECT) has been used for risk stratification before non-cardiac surgery. However, few authors have used mathematical models for evaluating the likelihood of perioperative cardiac events.

Methods and Results This retrospective cohort study collected data of 1,351 patients referred for SPECT before non-cardiac surgery. We generated binary classifiers using support vector machine (SVM) and conventional linear models for predicting perioperative cardiac events. We used clinical and surgical risk, and SPECT findings as input data, and the occurrence of all and hard cardiac events as output data. The area under the receiver-operating characteristic curve (AUC) was calculated for assessing the prediction accuracy. The AUC values were 0.884 and 0.748 in the SVM and linear models, respectively in predicting all cardiac events with clinical and surgical risk, and SPECT variables. The values were 0.861 (SVM) and 0.677 (linear) when not using SPECT data as input. In hard events, the AUC values were 0.892 (SVM) and 0.864 (linear) with SPECT, and 0.867 (SVM) and 0.768 (linear) without SPECT.
\end{abstract}

Conclusion The SVM was superior to the linear model in risk stratification. We also found an incremental prognostic value of SPECT results over information about clinical and surgical risk. (Circ J 2008; 72: 1829-1835)

Key Words: Gated single-photon emission computed tomography; Myocardial perfusion; Perioperative cardiac event; Perioperative risk stratification; Support vector machine

$\mathbf{C}$ ardiac events are important causes of perioperative mortality and morbidity in non-cardiac surgery. Thus, cardiac risk should be stratified in individual patients who are to undergo surgery, but it is difficult to assess the likelihood of perioperative cardiac events mainly because of complicated interrelationships between clinical risk factors and type of surgery. To overcome this problem, The American College of Cardiology/American Heart Association (ACC/AHA) Task Force published guidelines for perioperative cardiovascular evaluation for non-cardiac surgery in 1996 and 2002!,2 These guidelines divide clinical risk factors into major, intermediate and minor categories, and surgical procedures into high, intermediate and low-risk types. These are then used to determine further preoperative examinations, preoperative therapy, operative performance and perioperative management. The guidelines recommend non-invasive cardiac testing including myocardial perfusion imaging to be used mainly in patients with poor functional capacity ( $<4$ METs). Although there have been enough data

(Received March 14, 2008; revised manuscript received June 22, 2008; accepted June 24, 2008; released online September 24, 2008) Department of Radiology, School of Medicine, Keio University, *Department of Electrical Informatics, Hosei University and **21st Century Center of Excellence Program, Department of Radiology, School of Medicine, Keio University, Tokyo, Japan

Mailing address: Tomotaka Kasamatsu, MD, Department of Radiology, School of Medicine, Keio University, 35 Shinanomachi, Shinjukuku, Tokyo 160-8582, Japan. E-mail: kasa76@yahoo.co.jp

All rights are reserved to the Japanese Circulation Society. For permissions, please e-mail: cj@j-circ.or.jp concerning the use of myocardial perfusion single-photon emission computed tomography (SPECT) for preoperative risk stratification in high-risk surgery, ${ }^{3-17}$ little and only 2 studies are available about intermediate- $11,13,17-21$ and lowrisk surgery ${ }^{17,19}$ respectively.

Some investigators used mathematical models to provide the likelihood of perioperative cardiac events on the basis of clinical risk and SPECT findings! 10,22 They used a Bayesian model $^{10}$ or an artificial neural network ${ }^{22}$ as a tool to calculate the expected cardiac event rates. Advantages of the Bayesian model are that it permits consideration of the procedurespecific institutional complication rate in the estimation of risk and that it is not necessary to assume independence between variables included in the model $1^{10}$ The artificial neural network generally allows lower computational burden and is reported to be more accurate in predicting cardiac events for high-risk patients compared with a logistic regression model ${ }^{22}$ These reports focused on risk stratification of patients undergoing high-risk surgery, and risk of other types of surgery was not evaluated.

Recently, number of researchers used support vector machine (SVM)-based classifiers in the field of language processing, biology and so on. ${ }^{23-26}$ The SVM is a kind of supervised learning method that yields an appropriate discrimination program by using data whose inputs and accurate outputs are already known (training dataset). It first generates an $\mathrm{N}$-dimensional hyperplane that best separates the training data into 2 different half spaces, and then classify unknown de novo data by determining the half space they belong to. We introduced a SVM in preoperative risk strati- 
Table 1 Surgical Risk and Cardiac Events

\begin{tabular}{lccc}
\hline \hline Surgical risk grade & No. of patients & All cardiac event & Hard event \\
\hline High-risk & 511 & $35(6.8 \%)$ & $10(2.0 \%)$ \\
Intermediate-risk & 572 & $35(6.1 \%)$ & $3(0.5 \%)$ \\
Low-risk & 268 & $6(2.2 \%)$ & $1(0.4 \%)$ \\
Total & 1,351 & $76(5.6 \%)$ & $14(1.0 \%)$ \\
\hline
\end{tabular}

Table 2 Clinical Characteristics of 1,351 Patients

\begin{tabular}{lcccc}
\hline \hline Risk factor & $\begin{array}{c}\text { No. of patients with } \\
\text { each risk factor }\end{array}$ & $\begin{array}{c}\text { Event rate in patients } \\
\text { with each risk factor }\end{array}$ & Relative risk & $p$ value \\
\hline Aged 75 or more & $404(30 \%)$ & $27 / 404(6.7 \%)$ & 1.3 & $N S$ \\
Sex (male) & $965(71 \%)$ & $57 / 965(5.9 \%)$ & 1.2 & $N S$ \\
Hypertension & $577(43 \%)$ & $37 / 577(6.4 \%)$ & 1.3 & $N S$ \\
Diabetes & $277(21 \%)$ & $17 / 277(6.1 \%)$ & 1.1 & $N S$ \\
History of myocardial infarction & $180(13 \%)$ & $18 / 180(10 \%)$ & 2.0 & $<0.05$ \\
Chest pain & $208(15 \%)$ & $15 / 208(7.2 \%)$ & 1.4 & $N S$ \\
History of HF & $62(4.6 \%)$ & $13 / 62(21 \%)$ & 4.3 & $<0.0001$ \\
History of revascularization & $104(7.7 \%)$ & $11 / 104(11 \%)$ & 2.0 & $<0.05$ \\
ECG arrhythmia & $204(15 \%)$ & $16 / 204(7.8 \%)$ & 1.5 & $N S$ \\
ECG Q-wave & $237(18 \%)$ & $21 / 237(8.9 \%)$ & 1.8 & $<0.05$ \\
ECG ST change & $453(34 \%)$ & $36 / 453(7.9 \%)$ & 1.8 & $<0.05$ \\
SRS $\geq 4$ & $241(18 \%)$ & $28 / 241(12 \%)$ & 2.7 & $<0.0001$ \\
SSS $\geq 4$ & $331(25 \%)$ & $37 / 331(11 \%)$ & 2.9 & $<0.0001$ \\
SDS $\geq 4$ & $146(11 \%)$ & $18 / 146(12 \%)$ & 2.6 & $<0.001$ \\
QGS abnormality & $378(28 \%)$ & $49 / 378(13 \%)$ & 4.7 & $<0.0001$ \\
\hline
\end{tabular}

$H F$, heart failure; ECG, electrocardiography; SRS, summed rest score; SSS, summed stress score; SDS, summed difference score; $Q G S$, quantitative gated-single-photon emission computed tomography (SPECT).

fication in the present study. In this context, the above 2 half spaces equal to the 2 situations: the presence and absence of cardiac events, and the training dataset consists of data of each patient whose predictive variables and the prognostic outcome are already known. The hyperplane is determined by a cluster of data located near the border of the 2 spaces, which are called support vectors. The SVM analysis selects the appropriate hyperplane to maximize the margin between the hyperplane and support vectors. Among supervised learning methods, SVM is thought to be one of the most accurate techniques. However, as far as we investigated, there seems to be only 1 report using SVM for the risk assessment of patients with cardiac diseases ${ }^{26}$ and no data is available using SVM for stratifying perioperative cardiac risk.

In the current study, we generated linear and SVM classifiers, and evaluated their accuracies in predicting perioperative cardiac events in all types of non-cardiac surgery including high, intermediate and low-risk procedures. Furthermore, we estimated an incremental prognostic value of SPECT indices in each model.

\section{Methods}

\section{Study Design}

This retrospective cohort study used data collected from patients referred for preoperative stress myocardial perfusion SPECT before non-cardiac surgery. Written informed consent was obtained from all patients concerning the examination protocol, possible side-effects and the use of the data for research purposes. The form to obtain consent was approved by the institutional review committee. This study was also conducted according to the governmental and institutional regulations for protection of data privacy and confidentiality.

\section{Patient Population}

Our database for preoperative risk stratification with myocardial perfusion SPECT was used to identify 1,517 consecutive patients who underwent preoperative dipyridamole stress SPECT with electrocardiography (ECG)-gating between October 1999 to December 2006. Operation was cancelled or deferred after SPECT imaging in 166 patients, and 1,351 patients underwent surgery and were analyzed. Of the former 166, 74 underwent coronary revascularization and surgery was cancelled in 92 . All of the 166 patients were excluded from data analyses.

As indicated in Table 1, all the surgical procedures were classified according to the guidelines of ACC/AHA 1,2 Table 1 also includes the cardiac event rates in each subgroup classified by surgical risk. Cardiac events developed in $76(5.6 \%)$ of the 1,351 patients, and hard events occurred in $14(1.0 \%)$. Hard events include 9 cardiac deaths and 5 non-fatal myocardial infarction. Two of the 5 patients with myocardial infarction also manifested subsequent heart failure. Besides the above 14 patients, 19 and 43 had unstable angina and heart failure, respectively. Demographic data of the patients including clinical risk factors and scintigraphic findings are listed in Table2.

\section{Myocardial Perfusion Imaging}

Technetium-99m tetrofosmin or Sestamibi was the imaging agent for myocardial perfusion imaging. A rest/stress 1-day protocol was applied using doses of $300 / 850 \mathrm{MBq}$ of tracer, and SPECT acquisition was started $30 \mathrm{~min}$ after injection. In the stress study, patients were continuously injected with $0.56 \mathrm{mg} / \mathrm{kg}$ of dipyridamole for $4 \mathrm{~min}$, and then the perfusion tracer was administered $3 \mathrm{~min}$ after the completion of dipyridamole infusion. Myocardial perfusion and cardiac function were simultaneously assessed by ECGgating. 
Table 3 Predictors and Their Conversion to Input Variables

\begin{tabular}{|c|c|c|}
\hline Classification & Predictor & Input \\
\hline Ope risk $(I C-1, I C-2)$ & Ope risk & High (3), intermediate (2), low (1) \\
\hline \multirow[t]{13}{*}{ Clinical factor (IC-1, IC-2) } & Age & Real age \\
\hline & Aged 75 or more & Aged 75 or more $(1)$, others $(0)$ \\
\hline & Sex & Male (1), female (0) \\
\hline & Hypertension & Yes (1), no (0) \\
\hline & Diabetes $^{\dagger}$ & Yes (1), no (0) \\
\hline & History of myocardial infarction ${ }^{\dagger}$ & Yes (1), no (0) \\
\hline & Chest pain & Yes (1), no (0) \\
\hline & History of $\mathrm{HF}^{\dagger}$ & Yes (1), no (0) \\
\hline & History of revascularization & Yes (1), no (0) \\
\hline & ECG arrhythmia & Yes (1), no (0) \\
\hline & $E C G Q$-wave & Yes (1), no (0) \\
\hline & ECG ST change & $\operatorname{Yes}(1)$, no $(0)$ \\
\hline & Clinical risk* & Yes*(2), no (1) \\
\hline \multirow[t]{3}{*}{ SPECT (perfusion) (IC-1) } & SRS & 17 segments total score \\
\hline & SSS & 17 segments total score \\
\hline & $S D S$ & 17 segments total score \\
\hline \multirow[t]{3}{*}{ SPECT (cardiac function) (IC-1) } & QGS abnormality & Yes (1), no $(0)$ \\
\hline & $\operatorname{LVEF}(\%)$ & 100-LVEF\% \\
\hline & No. of hypokinetic segments & $0-7$ \\
\hline
\end{tabular}

IC-1 and IC-2 denote input candidates with and without SPECT variables, respectively* patients with any of the 4 predictors ${ }^{\dagger}$ are assigned 2 as clinical risk.

LVEF, left ventricular ejection fraction. Other abbreviations see in Table 2.

A 3-headed rotating gamma camera Toshiba GCA9300A/DI (Toshiba Corporation, Tokyo, Japan) was used for data acquisition and a medical image processor GMS5500U/DI (Toshiba Corporation) was used for image processing. The gamma camera rotated for 15 min per acquisition. SPECT images were reconstructed into a $128 \times 128$ matrix with a ramp filter after processing 90 projections over 360-degrees using a Butterworth filter (order 8, cutoff 0.16 cycles/pixel). Gated-SPECT acquisition was implemented by dividing the cardiac cycle into 10 periods. Image data was resized to a $64 \times 64$ matrix before gated data analysis.

Rest and stress SPECT images were semi-quantitatively assessed using 4-point scoring scale (0: normal, 1: mild hypoperfusion; 2: moderate and severe hypoperfusion, and 3: perfusion defect, visual assessment) in the following 17 myocardial segments: 6 segments in a basal short-axis image, 6 segments in a mid short-axis image, 4 segments in a apical short-axis slice and 1 apical segment in a long-axis image 19,27 The summed rest score (SRS) and summed stress scores (SSS) were defined as the total of the scores in the 17 segments from rest and stress images, respectively. The summed difference score (SDS) was calculated by subtracting the SRS from the SSS.

Quantitative gated-SPECT (QGS) program was used for gated-SPECT analysis ${ }^{28}$ Calculation of the left ventricular ejection fraction (LVEF) value was performed by means of automatic determination of endocardial and epicardial surfaces for all gating intervals in the cardiac cycle. We used stress LVEF because post-stress data includes the influence of ischemic stunning 29 which might provide an additional prognostic value. In addition, regional wall motion was visually assessed through the cine-mode display of the QGS program by dividing the myocardium into 7 segments according to the AHA criteria: anterobasal, anterolateral, apical, inferior, posterobasal, septal and posterolateral. We recorded the number of hypokinetic segments. The presence of QGS abnormality was defined as the presence of 1 or more hypokinetic segments. We considered not only re- gional wall motion but also wall thickening in assessing wall motion in the septum? 30

\section{Medical Record Review}

We assessed the occurrence of cardiac events by reviewing the medical record. Perioperative cardiac events were defined as those that occurred during, and within 1 month after surgery? Cardiac events included cardiac death, nonfatal myocardial infarction, unstable angina and congestive heart failure defined as follows, ${ }^{8}, 10$ Cardiac death was defined as death as a result of myocardial infarction, heart failure or arrhythmias. The diagnostic criteria for myocardial infarction was based on changes of ECG and the serum creatine kinase value: new ECG Q-wave $>1 \mathrm{~mm}$ or creatine kinase myocardial-bound $>5 \%$ or both. Unstable angina was defined as electrocardiographic ST changes (ST-segment depression or elevation of $>1 \mathrm{~mm}$ on 12-lead ECG) with cardiac symptoms. Congestive heart failure was defined as radiographic evidence of pulmonary edema and cardiac enlargement requiring inotropic support. Non-fatal arrhythmias were excluded from cardiac events. Hard events comprised cardiac death and non-fatal myocardial infarction.

\section{Variable Selection}

A total of 20 variables were used in the model creation and analysis (Table 3 ). These 20 consisted of 1 parameter reflecting surgical risk, 13 clinical risk factors and 6 scintigraphic indices (3 perfusion and 3 functional variables). Before input into the model, each parameter was normalized to have the mean value 0 and the standard deviation 1 .

\section{Model Development}

We generated binary classifiers for predicting perioperative cardiac events using a SVM or a linear model with the above 20 input variables, and evaluated their predictive accuracies? 23,24 We used SVM light (free software) for the SVM model, and SPSS v14 (SPSS, Chicago, IL, USA) and MATLAB v13 (MathWorks, Natick, MA, USA) for the 
Table 4 Predicting All Cardiac Events

\begin{tabular}{|c|c|c|c|c|c|c|c|c|c|}
\hline Classifier & $\begin{array}{l}\text { Candidate } \\
\text { for input }\end{array}$ & Selected input & $\begin{array}{l}\text { Sensitivity } \\
(\%)\end{array}$ & $\begin{array}{l}\text { Specificity } \\
(\%)\end{array}$ & $A U C$ & No. of SV & $C$ & $\sigma$ & $r$ \\
\hline \multirow[t]{2}{*}{$S V M$} & $I C-1$ & $\begin{array}{l}\text { Operation risk, clinical risk, history of } H F, \\
\text { ECG ST change, QGS abnormality }\end{array}$ & 97.4 & 72.2 & 0.884 & $304(22.5 \%)$ & 0.001 & 0.1 & 3 \\
\hline & $I C-2$ & $\begin{array}{l}\text { Operation risk, clinical risk, history of } H F, \\
\text { ECG ST change }\end{array}$ & 85.5 & 76.5 & 0.861 & $322(23.8 \%)$ & 0.1 & 1 & 3 \\
\hline \multirow[t]{2}{*}{ Linear } & $I C-1$ & $\begin{array}{l}\text { Age, operation risk, history of } H F \\
Q G S \text { abnormality }\end{array}$ & 67.1 & 75.4 & 0.748 & - & - & - & - \\
\hline & $I C-2$ & $\begin{array}{l}\text { Operation risk, clinical risk, history of } H F \text {, } \\
\text { ECG ST change }\end{array}$ & 72.4 & 62.4 & 0.677 & - & - & - & - \\
\hline
\end{tabular}

$A U C$, area under the ROC curve; SV, support vectors; $C$, soft margin; $\sigma$, standard deviation of Gaussian kernel function; $r$, error risk factor. Other abbreviations see in Table 2.

Table 5 Predicting Hard Events

\begin{tabular}{|c|c|c|c|c|c|c|c|c|c|}
\hline Classifier & $\begin{array}{l}\text { Candidates } \\
\text { for input }\end{array}$ & Selected input & $\begin{array}{l}\text { Sensitivity } \\
(\%)\end{array}$ & $\begin{array}{l}\text { Specificity } \\
(\%)\end{array}$ & $A U C$ & No. of SV & $C$ & $\sigma$ & $r$ \\
\hline \multirow[t]{2}{*}{$S V M$} & $I C-1$ & $\begin{array}{l}\text { Operation risk, history of revascularization, } \\
E C G Q \text { wave, } S R S, Q G S \text { abnormality }\end{array}$ & 92.9 & 86.2 & 0.892 & $298(22.1 \%)$ & 0.001 & 3 & 20 \\
\hline & $I C-2$ & $\begin{array}{l}\text { Operation risk, history of revascularization, } \\
\text { history of } H F, E C G Q \text { wave }\end{array}$ & 85.7 & 76.7 & 0.867 & $471(34.9 \%)$ & 1 & 5 & 5 \\
\hline \multirow[t]{2}{*}{ Linear } & $I C-1$ & $\begin{array}{l}\text { Operation risk, history of revascularization, } \\
\text { ECG } Q \text { wave, } S R S, Q G S \text { abnormality }\end{array}$ & 85.7 & 81.5 & 0.864 & - & - & - & - \\
\hline & $I C-2$ & $\begin{array}{l}\text { Operation risk, clinical risk, history of } H F \text {, } \\
\text { ECG ST change }\end{array}$ & 71.4 & 79.4 & 0.768 & - & - & - & - \\
\hline
\end{tabular}

Abbreviations see in Tables 2,4.

linear model. As for the output of the classifiers, we assigned 1 and -1 to the presence and absence of cardiac events, respectively. In the SVM, we used a Gaussian kernel to improve the predictive accuracy and reduce the computational burden in non-linear classification. ${ }^{25}$ A Gaussian kernel SVM requires the following 3 parameters to determine the hyperplane: $\mathrm{C}$ (soft margin), $\sigma$ (standard deviation of Gaussian kernel function) and $\mathrm{r}$ (error risk factor). We generated a series of SVM models to select the model providing the best predictive performance. In the model creation, we changed the 3 parameters within the following ranges: $0.001 \leq \mathrm{C} \leq 20,0.001 \leq \sigma \leq 10,1 \leq \mathrm{r} \leq 20$, and identified the most significant predictive variable(s) among the 20 through an incremental stepwise method with a hypothesis test of Wilks' lambda to search appropriate input parameters ${ }^{31}$ The linear classifier also generates a hyperplane that separates the dataset into 2 different half spaces. The hyperplane is determined by a linear combination of predictive variables. The linear model used the same training data and input selection method as well as the SVM one. In each input-selection step, a statistical F-test is carried out and the predictive variable with the highest partial correlation coefficient under $\mathrm{p}<0.05$ is selected while a statistically negligible $(p>0.10)$ variable is rejected. This process was repeated until no further input satisfied the above-mentioned criteria. As indicated in Table 3, we generated discrimination models with the above 20 input variables (input candidate-1, IC-1) and 14 inputs not including 6 SPECT variables (input candidate-2, IC-2), and compared their predictive accuracies to assess an incremental prognostic value of SPECT results.

\section{Evaluation and Statistics}

Fisher's exact test was used in univariate analysis to compare the frequency of cardiac events between patients with positive and negative test results or risk factors. A p-value of $<0.05$ was considered statistically significant in the analysis.

We obtained sensitivity, specificity and the area under the receiver-operating characteristic curve (AUC) to evaluate the predictive performance of the classifiers using leaveone-out cross validation (LOOCV) 32 First, LOOCV removes a single data as the validation data from the original dataset and uses the remaining data as the training dataset. The single data is input into the classifier generated by using the remaining data to judge whether the classification is correct. This judgment is repeated such that each data in the original dataset is used once as the validation data.

\section{Results}

Clinical Risk Factors Predictive of Cardiac Events

Table 2 shows the characteristics of the patients and the results of univariate analysis for seeking variables predicting perioperative cardiac events. The history of myocardial infarction, heart failure or revascularization, Q-wave or ST change in ECG, and SPECT findings including the SRS, SSS and SDS, and QGS abnormality presented significant risk stratification.

\section{Predicting All Cardiac Events}

Table 4 shows selected input variables and the accuracy in predicting all cardiac events. Among the 20 variables, all classifiers selected operation risk and the history of heart failure as input. Three of the 4 selected clinical risk and the ST change in ECG. Aging was selected only by linear IC-1. Regarding the scintigraphic variables, only QGS abnormality was selected by the 2 classifiers with IC-1. The SVM models yielded favorable predictive performance in terms of the AUC value compared with the linear models regardless of the use of SPECT results. The incremental prognostic value of SPECT was considerable in the linear model, 
meanwhile it was marginal in the SVM (comparing IC-1 and IC-2 in each model in Table4).

\section{Predicting Hard Events}

The results in predicting hard events are shown in Table 5. Operation risk was also introduced by all classifiers. The Q-wave in ECG and the history of revascularization were the most frequently chosen clinical risk factors followed by the history of heart failure. Among the scintigraphic variables, QGS abnormality and the SRS were selected. The SVM models also provided better results in predicting hard events than the linear models, but the difference was not so marked compared with predicting all cardiac events. The predictive performance of the linear model was moderately improved by adding SPECT indices, in contrast, the improvement was modest in the SVM model.

\section{Discussion}

Myocardial perfusion imaging has gained a widespread acceptance in the assessment of cardiac risk ${ }^{33-35}$ In the current study, we generated discrimination models using SVM, and evaluated their accuracies and an incremental value of myocardial perfusion imaging in predicting perioperative cardiac events in high, intermediate and low-risk non-cardiac surgery. The SVM offered improved predictive accuracy of perioperative cardiac events compared with the conventional linear model, and the prognostic utility of gated SPECT was also confirmed.

In the field of medicine, the SVM has mainly been used for classifying tumor cells into benign or malignant, and predicting the subcellular localization of proteins using the amino acid sequence. There seems to be only 1 report using the SVM for cardiac risk stratification ${ }^{26}$ in which the SVM classifiers offered more favorable results compared with others. The SVM affords a complex hyperplane by using support vectors and maximization of the margin, even when a linear separation is difficult or impossible. It can incorporate a complex dataset in which some pieces of data have the same or similar inputs and different outputs. In the current study, because cardiac events occur only in a limited part of the patients, the training dataset includes a certain number of patients with the same or similar preoperative predictive variables and different prognostic outcome. This situation degrades the predictive accuracy of the classifiers in perioperative cardiac risk stratification. To overcome this problem, we used a SVM-based classifier that enables us to define a complex hyperplane to separate patients into different prognostic groups instead of a conventional linear model. In the current study, the SVM model offered favorable results compared with the linear model in terms of the AUC value, suggesting the feasibility of this technique. This model might be applicable not only to the binary classification but also to providing the likelihood of cardiac events by measuring the distance between the hyperplane and the point in the $\mathrm{N}$-dimensional space determined by the input dataset of each patient.

The frequency of hard events was very low (1.0\%) compared with that of all events (5.6\%). However, as indicated in Tables 4 and 5, the numbers of support vectors determining the hyperplane were 304 and 298 (about 22\% of the total of the training dataset) in models predicting all and hard cardiac events, respectively, indicating that the both SVM classifiers yielded comparably robust hyperplanes. Concerning the number of input variables and the event frequency, it is ideal to obtain high predictive accuracy by using a small number of input variables in predicting events of very low frequency. However, the accuracy decreased when using input variables less than 5 in the present study (data not shown). Because we selected the 5 variables using the hypothesis test of Wilks' Lambda as mentioned in the materials and method section, the 5 are statistically significant for prediction. In addition, selected variables dispersed over the categories of operation risk, clinical risk factors and SPECT results, suggesting the interrelationships among variables are not so close. Moreover, it is thought that the LOOCV method used for the evaluation is a powerful technique to apply to a cohort that has a low frequency of events ${ }^{36}$ As the predictive accuracies obtained with 5 variables were good both in the SVM and linear models, we think that the classifiers have acceptable reliability even in predicting events of low frequency.

The predictive performance of the conventional linear models was improved by adding SPECT indices, indicating its incremental prognostic value. Although the improvement was modest in the SVM models, the number of support vectors decreased after adding SPECT results, suggesting that the classification became stable. The clinical significance of ECG-gated acquisition was verified by the fact that QGS abnormality was selected in all IC-1 models as input. We calculated the AUC values when using input variables with and without the SRS in IC-1 to compare the prognostic value of the SRS and QGS abnormality. Although there was a certain difference in the AUC value between IC-1 and IC-2 in the linear model (0.864 and 0.768), the incremental prognostic value of the SRS was not marked in IC-1 (the AUC was 0.838 when not using the SRS). This result suggests that the prognostic significance of the SRS was limited compared with the QGS abnormality in the current study. This situation is the same in the SVM model because there was a little difference of the AUC value between IC-1 and IC-2 in the SVM. In addition, the SSS and SDS were not selected as input variables by the classifiers. A certain amount of patients with high values of the SSS or SDS (related to stress-induced ischemia) were excluded from the analyses because of the cancellation of surgery or performance of preoperative coronary revascularization (These patients were included in the 166 patients whose surgery was cancelled or deferred). Therefore, it is thought that the SSS and SDS were not significant predictors in the current study. Further studies are required to assess the prognostic value of the above perfusion indices by increasing the number of patients who manifested moderate or severe ischemia in SPECT and underwent surgery without preoperative revascularization.

As mentioned above, the SVM is an excellent tool for data classification based on supervised learning, although it is not very popular in medical practice. We introduced the SVM to preoperative risk stratification with SPECT and showed its superiority to the conventional linear model. This technique is thought to be applicable not only to predicting perioperative cardiac events but also to cardiac risk assessment in general situations, and to the evaluation of an incremental prognostic value of a specific cardiac examination. The SVM is expected to be widely used for a medical research in the future.

\section{Study Limitations}

There are some limitations in the present study. First, surgeons and anesthesiologists were not blinded to the results 
of cardiac testing. This availability of results had an influence on decisions regarding perioperative management of patients, which could lower the cardiac event rate and underestimate the predictive accuracy of the models. Next, we analyzed the data obtained from patients who underwent preoperative SPECT. Most of them were referred for SPECT because of concern about perioperative cardiac risk. Thus, these patients do not epitomize the general population of non-cardiac surgery candidates in terms of the likelihood of cardiac events. In addition, event rates in patients undergoing low-risk surgery were very low even if they have some cardiac risk. It is inherently difficult to specify highrisk patients in this cohort regardless of classifiers used. Further study is required to assess the predictive performance of these models in cohorts without particular cardiac risk and/or undergoing low-risk surgery.

\section{Conclusion}

In summary, the superiority of SVM was elucidated in assessing the likelihood of perioperative cardiac events compared with the conventional linear model. We also found an incremental prognostic value of SPECT results over information about clinical and surgical risk.

\section{Acknowledgements}

This study is partially supported by Grant-in-Aid for Scientific Research (18790912) from the Ministry of Education, Culture, Sports, Science and Technology.

\section{References}

1. Eagle KA, Brundage BH, Chaitman BR, Ewy GA, Fleisher LA, Hertzer NR, et al. Guidelines for perioperative cardiovascular evaluation for noncardiac surgery: Report of the American College of Cardiology/American Heart Association Task Force on Practice Guidelines. J Am Coll Cardiol 1996; 27: 910-948.

2. Eagle KA, Berger PB, Calkins H, Chaitman BR, Ewy GA, Fleischmann KE, et al; American College of Cardiology; American Heart Association. ACC/AHA guideline update for perioperative cardiovascular evaluation for noncardiac surgery-executive summary: A report of the American College of Cardiology/American Heart Association Task Force on Practice Guidelines (Committee to Update the 1996 Guidelines on Perioperative Cardiovascular Evaluation for Noncardiac Surgery). J Am Coll Cardiol 2002; 39: 542-553.

3. Klonaris CN, Bastounis EA, Xiromeritis NC, Balas PE. The predictive value of dipyridamole-thallium scintigraphy for cardiac risk assessment before major vascular surgery. Int Angiol 1998; 17: 171-178.

4. Huang Z, Komori S, Sawanobori T, Kohno I, Sano S, Ishihara T, et al. Dipyridamole thallium-201 single-photon emission computed tomography for prediction of perioperative cardiac events in patients with arteriosclerosis obliterans undergoing vascular surgery. Jpn Circ J 1998; 62: 274-278.

5. Chen T, Kuwabara Y, Tsutsui H, Sasaki M, Nakagawa M, Koga H, et al. The usefulness of dipyridamole thallium-201 single photon emission computed tomography for predicting perioperative cardiac events in patients undergoing non-cardiac vascular surgery. Ann Nucl Med 2002; 16: 45-53.

6. Harafuji K, Chikamori T, Kawaguchi S, Obitsu Y, Ito S, Igarashi Y, et al. Value of pharmacologic stress myocardial perfusion imaging for preoperative risk stratification for aortic surgery. Circ J 2005; 69: $558-563$.

7. Stratmann HG, Younis LT, Wittry MD, Amato M, Miller DD. Dipyridamole technetium-99m sestamibi myocardial tomography in patients evaluated for elective vascular surgery: Prognostic value for perioperative and late cardiac events. Am Heart J 1995; 131: $923-$ 929.

8. Vanzetto G, Machecourt J, Blendea D, Fagret D, Borrel E, Magne JL, et al. Additive value of thallium single-photon emission computed tomography myocardial imaging for prediction of perioperative events in clinically selected high cardiac risk patients having abdominal aortic surgery. Am J Cardiol 1996; 77: 143-148.

9. Kertai MD, Boersma E, Bax JJ, Heijenbrok-Kal MH, Hunink MG,
L'talien GJ, et al. A meta-analysis comparing the prognostic accuracy of six diagnostic tests for predicting perioperative cardiac risk in patients undergoing major vascular surgery. Heart 2003; 89: 1327 1334.

10. L'Italien GJ, Paul SD, Hendel RC, Leppo JA, Cohen MC, Fleisher LA, et al. Development and validation of a Bayesian model for perioperative cardiac risk assessment in a cohort of 1,081 vascular surgical candidates. J Am Coll Cardiol 1996; 27: 779-786.

11. Rubin DN, Ballal RS, Marwick TH. Outcomes and cost implications of a clinical-based algorithm to guide the discriminate use of stress imaging before noncardiac surgery. Am Heart J 1997; 134: 83-92.

12. Heiba SI, Jacobson AF, Shattuc S, Ferreira MJ, Sharma PN, Cerqueira MD. The additive values of left ventricular function and extent of myocardium at risk to dipyridamole perfusion imaging for optimal risk stratification prior to vascular surgery. Nucl Med Commun 1999; 20: $887-894$.

13. Hashimoto J, Suzuki T, Nakahara T, Kosuda S, Kubo A. Preoperative risk stratification using stress myocardial perfusion scintigraphy with electrocardiographic gating. J Nucl Med 2003; 44: 385-390.

14. Leppo J, Plaja J, Gionet M, Tumolo J, Paraskos JA, Cutler BS. Noninvasive evaluation of cardiac risk before elective vascular surgery. J Am Coll Cardiol 1987; 9: 269-276.

15. Palda AV, Detsky AS. Guidelines for assessing and managing the perioperative risk from coronary artery disease associated with major noncardiac surgery. Ann Intern Med 1997; 127: 309-312.

16. McPhail NV, Ruddy TD, Calvin JE, Davies RA, Barber GG. A comparison of dipyridamole-thallium imaging and exercise testing in the prediction of postoperative cardiac complications in patients requiring arterial reconstruction. J Vasc Surg 1989; 10: 51-56.

17. Bai J, Hashimoto J, Nakahara T, Kitamura N, Suzuki T, Kubo A. Preoperative risk evaluation in diabetic patients without angina. Diabetes Res Clin Pract 2008; 81: 150-154.

18. Chang K, Sarkiss M, Won KS, Swafford J, Broemeling L, Gayed I. Preoperative risk stratification using myocardial perfusion studies in patients with cancer. J Nucl Med 2007; 48: 344-348.

19. Hashimoto J, Nakahara T, Bai J, Kitamura N, Kasamatsu T, Kubo A. Preoperative risk stratification with myocardial perfusion imaging in intermediate and low risk non-cardiac surgery. Circ J 2007; 71: $1395-1400$

20. Takase B, Younis LT, Byers SL, Shaw LJ, Labovitz AJ, Chaitman BR, et al. Comparative prognostic value of clinical risk indexes, resting two dimensional echocardiography, and dipyridamole stress thallium201 myocardial imaging for perioperative cardiac events in major nonvascular surgery patients. Am Heart J 1993; 126: 1099-1106.

21. Bai J, Hashimoto J, Nakahara T, Suzuki T, Kubo A. Influence of ageing on perioperative cardiac risk in non-cardiac surgery. Age Ageing 2007; 36: 68-72.

22. Lapuerta P, L'Italien GJ, Paul S, Hendel RC, Leppo JA, Fleisher LA, et al. Neural network assessment of perioperative cardiac risk in vascular surgery patients. Med Decis Making 1998; 18: 70-75.

23. Cortes C, Vapnik V. Support-vector networks. Machine Learning 1995; 20: 273-297.

24. Burges CJC. A tutorial on support vector machines for pattern recognition. In: Fayyad U, editor. Data mining and knowledge discovery, vol.2. Boston: Kluwer Academic Publishers; 1998; 121-168.

25. Muller KR, Mika S, Ratsch G, Tsuda K, Scholkopf B. An introduction to kernel-based learning algorithms. IEEE Trans Neural Networks 2001; 2: 181-201.

26. Hammer PL, Bonates T. Logical analysis of data: From combinatorial optimization to medical applications. Ann Op Res 2006; 1: 203-225.

27. Cerqueira MD, Weissman NJ, Dilsizian V, Jacobs AK, Kaul S, Laskey WK, et al. Standardized myocardial segmentation and nomenclature for tomographic imaging of the heart: A statement for healthcare professionals from the Cardiac Imaging Committee of the Council on Clinical Cardiology of the American Heart Association. Circulation 2002; 105: 539-542.

28. Germano G, Kiat H, Kavanagh PB, Moriel M, Mazzanti M, Su H, et al. Automatic quantification of ejection fraction from gated myocardial perfusion SPECT. J Nucl Med 1995; 36: 2138-2147.

29. Hashimoto J, Kubo A, Iwasaki R, Iwanaga S, Mitamura H, Ogawa S. Gated single-photon emission computed tomography imaging protocol to evaluate myocardial stunning after exercise. Eur J Nucl Med 1999; 26: $1541-1546$.

30. Higuchi T, Nakajima K, Taki J, Tsuji S, Tonami N. Assessment of regional myocardial function by ECG-gated myocardial SPECT. Kaku-Igaku 1999; 36: 445-451.

31. Everitt BS, Dunn G. Applied multivariate data analysis. London: Edward Arnold; 1991; 219-220.

32. Shao J. Linear model selection by cross-validation. J Am Statis Assoc 1993; 88: 486-494. 
33. Nagao T, Chikamori T, Hida S, Igarashi Y, Kuwabara Y, Nishimura S, et al. Quantitative gated single-photon emission computed tomography with $(99 \mathrm{~m}) \mathrm{Tc}$ sestamibi predicts major cardiac events in elderly patients with known or suspected coronary artery disease: The QGSPrognostic Value in the Elderly (Q-PROVE) Study. Circ J 2007; 71: $1029-1034$

34. Matsumoto N, Sato Y, Suzuki Y, Kunimasa T, Yoda S, Iida J, et al Prognostic value of myocardial perfusion single-photon emission computed tomography for the prediction of future cardiac events in a
Japanese population: A middle-term follow-up study. Circ J 2007; 71: $1580-1585$.

35. Matsuo S, Nakajima K, Horie M, Nakae I, Nishimura T; The JACCESS Investigators. Prognostic value of normal stress myocardial perfusion imaging in Japanese population. Circ J 2008; 72: 611 617.

36. Efron B, Gong G. A leisurely look at the bootstrap, the jackknife, and cross-validation. Amer Statistician 1983; 37: 36-48. 